\title{
Genetic evidence for a causative effect of airflow obstruction on left ventricular filling: a Mendelian randomisation study
}

\author{
Lars Harbaum ${ }^{1 *}\left(\mathbb{D}\right.$, Jan K. Hennigs ${ }^{1}$, Marcel Simon ${ }^{1}$, Tim Oqueka ${ }^{1}$, Henrik Watz ${ }^{2}$ and Hans Klose ${ }^{1}$
}

\begin{abstract}
Background: Observational studies on the general population have suggested that airflow obstruction associates with left ventricular (LV) filling. To limit the influence of environmental risk factors/exposures, we used a Mendelian randomisation (MR) approach based on common genetic variations and tested whether a causative relation between airflow obstruction and LV filling can be detected.

Methods: We used summary statistics from large genome-wide association studies (GWAS) on the ratio of forced expiratory volume in $1 \mathrm{~s}$ to forced vital capacity (FEV1/FVC) measured by spirometry and the LV end-diastolic volume (LVEDV) as assessed by cardiac magnetic resonance imaging. The primary MR was based on an inverse variance weighted regression. Various complementary MR methods and subsets of the instrument variables were used to assess the plausibility of the findings.
\end{abstract}

Results: We obtained consistent evidence in our primary MR analysis and subsequent sensitivity analyses that reducing airflow obstruction leads to increased inflow to the LV (odds ratio [OR] from inverse variance weighted regression $1.05,95 \%$ confidence interval $[\mathrm{Cl}] 1.01-1.09, \mathrm{P}=0.0172$ ). Sensitivity analyses indicated a certain extent of negative horizontal pleiotropy and the estimate from biased-corrected MR-Egger was adjusted upward (OR 1.2, 95\% Cl 1.09-1.31, $P<0.001$ ). Prioritisation of single genetic variants revealed rs995758, rs2070600 and rs 7733410 as major contributors to the MR result.

Conclusion: Our findings indicate a causal relationship between airflow obstruction and LV filling in the general population providing genetic context to observational associations. The results suggest that targeting (even subclinical) airflow obstruction can lead to direct cardiac improvements, demonstrated by an increase in LVEDV. Functional annotation of single genetic variants contributing most to the causal effect estimate could help to prioritise biological underpinnings.

\section{Introduction}

Observational studies based on the general population have consistently demonstrated that increasing airflow obstruction can be associated with reduced left

\footnotetext{
*Correspondence: I.harbaum@uke.de

${ }^{1}$ Abteilung für Pneumologie, Centrum für Pulmonal Arterielle Hypertonie Hamburg (CPAHH), Universitätsklinikum Hamburg-Eppendorf, Hamburg, Germany

Full list of author information is available at the end of the article
}

ventricular (LV) filling [1-3]. The relationship between the ratio of forced expiratory volume in $1 \mathrm{~s}$ to forced vital capacity (FEV1/FVC) as a measure of airflow obstruction in pulmonary function tests and LV end-diastolic volumes (LVEDV) was nearly linear across the spectrum of lung function in the Multi-Ethnic Study of Atherosclerosis (MESA) Lung Study [1]. Similarly, a decline in FEV1/FVC ratio was associated with underfilling of the left heart in the longitudinal Coronary Artery Risk Development in Young Adults (CARDIA) study [2]. An original author(s) and the source, provide a link to the Creative Commons licence, and indicate if changes were made. The images or other third party material in this article are included in the article's Creative Commons licence, unless indicated otherwise in a credit line to the material. If material is not included in the article's Creative Commons licence and your intended use is not permitted by statutory regulation or exceeds the permitted use, you will need to obtain permission directly from the copyright holder. To view a copy of this licence, visit http://creativecommons.org/licenses/by/4.0/. The Creative Commons Public Domain Dedication waiver (http://creativeco mmons.org/publicdomain/zero/1.0/) applies to the data made available in this article, unless otherwise stated in a credit line to the data. 
increase of airflow obstruction has also been associated with cardiovascular outcomes in the general population. In the Copenhagen City Heart Study (CCHS) the FEV1/ FVC ratio was related to the risk of cardiovascular death independent of traditional cardiovascular risk factors [4]. These observations indicate that subclinical airflow obstruction may be linked with hemodynamic changes in the pulmonary circulation leading to reduced inflow to the LV.

Pulmonary and cardiac function variables are complex traits and large, population-based genome-wide association studies (GWAS) have provided multiple independent associations between genetic variation (mostly single-nucleotide polymorphism [SNP]) and quantitative measurements across the genome [5-8]. The principle of testing for an association of the same genetic variant with different traits allows to conclude on causality based on shared genetic risk. A Mendelian randomisation (MR) framework can help to provide such evidence and can be viewed as a meta-analysis of the causal estimates from each independent genetic variant [9]. Notably, since genetic variants are randomly allocated at conception, a MR approach limits the influence of environmental risk factors/exposures.

Using MR, we tested whether airflow obstruction may be casually linked to LV filling in large general population datasets.

\section{Methods}

\section{Selection of instruments for Mendelian randomisation}

In a two-sample Mendelian randomisation (MR) approach the instrument variables (IV) are developed from the association between common genetic variation and a risk factor or exposure (here FEV1/FVC) [10]. The outcome variable contains associations of these genetic variants with a second trait (here LVEDV) in an independent sample [10]. We used publicly available summary statistics (effect alleles, estimates and their standard errors) for SNPs that associated with FEV1/ FVC in a GWAS meta-analysis of the United Kingdom (UK) Biobank and SpiroMeta populations comprising up to 404,128 individuals to generate the IV (up to 321,047 individuals from the UK Biobank and 79,055 from the SpiroMeta consortium) [7]. We selected SNPs that associated with FEV1/FVC at genome-wide level of significance $\left(\mathrm{P}<5 \times 10^{-9}\right)$ [11] in the GWAS metaanalysis [7] and excluded palindromic variants (A/T or $\mathrm{C} / \mathrm{G}$ ) with intermediate allele frequencies (minor allele frequency $>45 \%$ ) to ensure that effects of variants for the exposure and outcome traits can be harmonized to the same allele. We retained common (minor allele frequency $[\mathrm{MAF}]>5 \%$ ) and uncorrelated SNPs (linkage disequilibrium $[\mathrm{LD}] \mathrm{r}^{2}<0.001$ within $\mathrm{a} \pm 10 \mathrm{Mb}$ window in the European 1000 Genomes phase 3 reference panel using PLINK 1.9 software), which were also available for the outcome. The outcome variable was obtained from a GWAS on LVEDV as measured by cardiac magnetic resonance imaging (MRI) in 36,042 individuals from the UK Biobank [5].

We tested for association of the IV with covariates querying all variants or proxies in high $\mathrm{LD}\left(\mathrm{r}^{2}>0.8\right)$ for association with disease endpoints or complex traits using a curated database of human genotype-phenotype associations (PhenoScanner V2) [12]. We did not consider association with pulmonary function test traits and intermediate phenotypes such as protein, metabolite or expression levels. To assess the influence on the causal estimate, we excluded variants that associated with one or more traits at genome-wide significance $\left(\mathrm{P}<5 \times 10^{-9}\right)$ followed by retesting.

To ensure that the overlap of individuals from the UK Biobank in the exposure and outcome cohorts did not overly influence our results (e.g., because of 'winner's curse' or overfitting) [13], we used a subset the IV including only SNPs that showed an association $\left(\mathrm{P}<1 \times 10^{-3}\right)$ in the SpiroMeta consortium population [14] and retested the causal estimate.

\section{Reverse Mendelian randomisation}

Applying identical data processing for a reverse MR analysis, we considered common and uncorrelated SNPs identified in the GWAS on LVEDV at genome-wide significance $\left(\mathrm{P}<5 \times 10^{-9}\right)$ as IV (exposure) [5]. The corresponding outcome variable was obtained from the GWAS meta-analysis of the UK Biobank and SpiroMeta populations on FEV1/FVC [7, 14]. Again, palindromic SNPs were excluded and only common and uncorrelated SNPs were retained.

\section{Statistical analyses}

The statistical approach of the MR strategy followed recent recommendations [15]. Primary MR analysis was performed using the inverse variance weighted (MRIVW) regression method under a multiplicative random-effects model using TwoSampleMR v0.5.4 software package in R-3.6.1 [16]. The MR-IVW method assumes no horizontal pleiotropy, where genetic variants independently associate with traits other than the ones under investigation, but will be unbiased if there is balanced pleiotropy.

In subsequent sensitivity analyses, we applied MR based on Egger regression (MR-Egger) [17] and weighted median (MR-WM) [18]. MR-Egger regression provides valid causal estimate when the InSIDE (Instrument Strength Independent of Direct Effect) assumption holds, even when all SNPs are invalid [17]. We used the adopted 
$\mathrm{I}^{2}$ statistic (referred to as $\mathrm{I}_{\mathrm{GX}}{ }_{\text {) }}$ ) to assess the NOME (no measurement error) assumption [19]. $\mathrm{I}_{\mathrm{GX}}^{2}$ provides an assessment of the degree of regression dilution due to uncertainty in the SNP-exposure associations and was proposed as a measure of instrument strength for the MR-Egger method [19]. MR-Egger intercept and funnel plots plotting the strength of variant associations with the exposure (as the inverse standard error) against the individual IV estimates were generated to assess whether pleiotropy is balanced or directional [17]. The MR-WM method has been proposed to complement MR-Egger in a sensitivity analyses as it does not to rely on the InSIDE assumption [18]. MR-WM provides valid causal estimates when $50 \%$ of the information is contributed from valid SNPs [18].

We additionally applied the MR-PRESSO (Mendelian Randomisation Pleiotropy RESidual Sum and Outlier) method to our MR model to identify and remove horizontal pleiotropic outlier variants followed by retesting for heterogeneity [20].

To assess the effects of individual SNPs, we performed single SNP MR analyses on each SNP individually using the simple ratio estimate (dividing the effect of the IV on the outcome by the effect of the IV on the exposure) and leave-one-out MR analyses, in which the causal estimate is retested using the MR-IVW regression method after sequentially omitting one SNP.

Findings at $\mathrm{P}<0.05$ were considered statistically significant.

\section{Results}

\section{Mendelian randomisation of FEV1/FVC on LVEDV}

Using 253 SNPs independently associated with FEV1/ FVC in a GWAS meta-analysis of UK Biobank and SpiroMeta consortium populations, we found genetic evidence for a causative effect of FEV1/FVC on LVEDV in our primary MR-IVW analysis with an odds ratio (OR) of 1.05 per increase of 1 standard deviation in FEV1/FVC (95\% confidence interval $[\mathrm{CI}] 1.01-1.09, \mathrm{P}=0.017$; Fig. 1 and Table 1). Directionality was consistent between Mendelian randomisation analysis and pervious observational data [1-3]. Full details on the variant filtering steps and IV can be found in the online supplement (Additional file 1: Fig. S1 and Additional file 2: Table S1).

The direction of effect estimates was consistent in subsequent sensitivity analyses (Table 1 ). The $\mathrm{I}^{2}{ }_{\mathrm{GX}}$ for the SNP-exposure associations was 0.99 indicating that attenuation due to NOME violation was small. A funnel plot displayed no asymmetry indicative for directional pleiotropy (Additional file 1: Fig. S2). The intercept from Egger-regression, however, suggested a certain extent of negative directional pleiotropy (Additional file 2: Table S2). Consequently, the bias-corrected MR-Egger

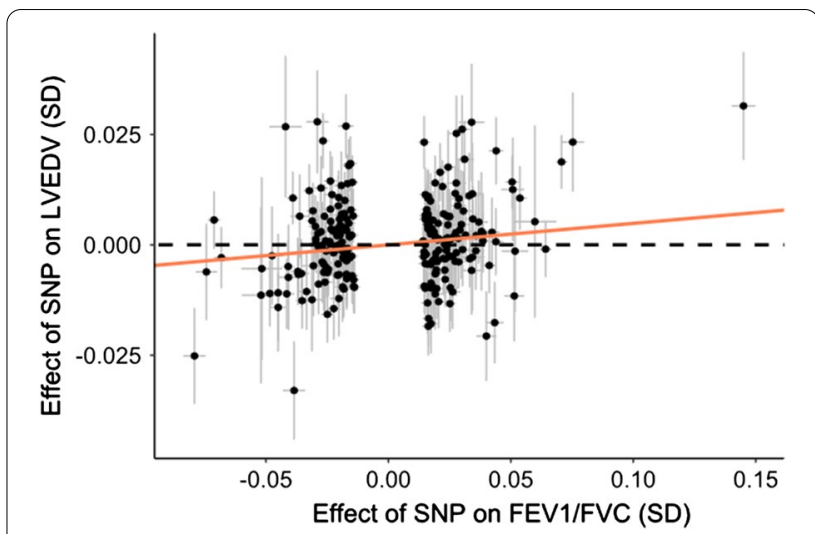

Fig. 1 Scatter plot of the effect estimates and standard error from 253 genetic variants on both FEV1/FVC and LVEDV. The orange line shows the overall causal effect from the inverse variance weighted regression

estimate was adjusted upward (OR 1.2, 95\% CI 1.09-1.31, $\mathrm{P}<0.001$; Table 1). The MR-WM provided a similar causal estimate as compared to the MR-IVW (OR 1.06, 95\% CI 1.01-1.12, $\mathrm{P}=0.028$; Table 1). There was evidence of heterogeneity in the SNP effect (Cochrane's Q 402) and MRPRESSO identified three influential outliers (rs4353138, rs7253302 and rs75928020) with an outlier test $\mathrm{P}=0.025$. With removal of these outliers, the causal effect estimate remained similar (OR 1.05, 95\% CI 1.02-1.10, $\mathrm{P}=0.007$; distortion test $\mathrm{P}=0.84$; Table 1$)$. We next performed leave-one-out MR-IVW analyses (excluding one variant at a time) and observed lower confidence interval ranging from 1 to 1.01 and upper from 1.08 to 1.1 (Additional file 2: Table S3).

To test for associations of the IV with covariates, we found that 107/253 variants associate with 291 different disease endpoints or other complex traits (Additional file 2: Table S4). With removal of these variants, a constant direction of effect estimates was observed in the primary MR-IVW (OR 1.09, 95\% CI 1.03-1.16, P =0.003) and subsequent sensitivity MR methods (Table 1). A funnel plot and the intercept from Egger-regression did not indicate directional pleiotropy, and MR-PRESSO did not identified outlier (Additional file 1: Fig. S2 and Table 2).

To test for an overestimation of the causal effect due to the overlap of individuals from the UK Biobank in the exposure and outcome cohorts, we selected 73/253 variants from the IV that showed association in SpiroMeta consortium population [14] and observed a constant direction of effect estimates in the primary MR-IVW (OR $1.06,95 \% \mathrm{CI} 1-1.12, \mathrm{P}=0.043)$ and in subsequent sensitivity analyses (Table 1). A funnel plot and the intercept of Egger-regression did not indicate directional pleiotropy (Additional file 1: Fig. S1 and Table 2). MR-PRESSO, however, identified rs75928020 as horizontal pleiotropic 
Table 1 Results of Mendelian randomisation (MR) analyses of FEV1/FVC on LVEDV

\begin{tabular}{|c|c|c|c|c|c|c|c|c|c|}
\hline \multirow{2}{*}{\multicolumn{2}{|c|}{ Instrument variables (IV) }} & \multirow[t]{2}{*}{ MR method } & \multirow[t]{2}{*}{ SNPs } & \multirow[t]{2}{*}{ Odds ratio } & \multicolumn{2}{|c|}{$\begin{array}{l}95 \% \\
\text { confidence } \\
\text { interval }\end{array}$} & \multirow[t]{2}{*}{ Estimate } & \multirow[t]{2}{*}{ Standard error } & \multirow[t]{2}{*}{ P-value } \\
\hline & & & & & Lower & Upper & & & \\
\hline \multirow{4}{*}{\multicolumn{2}{|c|}{ Full (primary IV) }} & MR-IVW & 253 & 1.05 & 1.01 & 1.09 & 0.05 & 0.02 & 0.017 \\
\hline & & MR-WM & 253 & 1.06 & 1.01 & 1.12 & 0.06 & 0.03 & 0.026 \\
\hline & & MR-Egger & 253 & 1.20 & 1.09 & 1.31 & 0.18 & 0.05 & $<0.001$ \\
\hline & & MR-PRESSO & 250 & 1.05 & 1.02 & 1.10 & 0.05 & 0.02 & 0.007 \\
\hline \multirow[t]{7}{*}{ Subset } & \multirow[t]{3}{*}{ No association to potential covariates } & MR-IVW & 146 & 1.07 & 1.01 & 1.13 & 0.06 & 0.03 & 0.019 \\
\hline & & MR-WM & 146 & 1.05 & 0.98 & 1.14 & 0.05 & 0.04 & 0.185 \\
\hline & & MR-Egger & 146 & 1.19 & 1.01 & 1.40 & 0.18 & 0.08 & 0.038 \\
\hline & \multirow{4}{*}{$\begin{array}{l}\text { Association with FEV1/FVC in the Spiro- } \\
\text { Meta consortium population }\end{array}$} & MR-IVW & 73 & 1.06 & 1.00 & 1.12 & 0.06 & 0.03 & 0.043 \\
\hline & & MR-WM & 73 & 1.05 & 0.97 & 1.13 & 0.05 & 0.04 & 0.214 \\
\hline & & MR-Egger & 73 & 1.12 & 0.98 & 1.28 & 0.12 & 0.07 & 0.091 \\
\hline & & MR-PRESSO & 72 & 1.06 & 1.01 & 1.12 & 0.06 & 0.03 & 0.021 \\
\hline
\end{tabular}

outlier variants (outlier test $\mathrm{P}=0.0073$ ). With removal, the causal effect remained similar (OR 1.06, 95\% CI 1.01$1.12, \mathrm{P}=0.021$; distortion test $\mathrm{P}=0.88$; Table 1 ).

To assess the relevance of individual SNPs, we performed single SNP MR analyses on each SNP individually and found that $141 / 253$ SNPs shared the direction of the overall MR effect, from which 19/141 were also nominally significant in single SNP MR analyses $(\mathrm{P}<0.05$; Table 2 and Additional file 2: Table S3). In leave-one-out MR-IVW analyses on these 19 SNPs, the causal effect estimate was reduced between 3 to $16 \%$ compared to the overall MR-IVW result (Table 2 and Additional file 2: Table S3).

\section{Reverse Mendelian randomisation}

To further understand a source of observational associations between FEV1/FVC and LVEDV in the general population, we performed the reversed MR experiment and used LVEDV-associated SNPs as instrument variable (exposure) to explore the relationship with FEV1/FVC (outcome). Using 13 SNPs independently associated with LVEDV, we found no evidence of an effect of LVEDV on FEV1/FVC using the primary MR-IVW (OR 1.02, 95\% CI $0.94-1.1, \mathrm{P}=0.62)$.

\section{Discussion}

Using a MR approach, we identified a causal relationship between FEV1/FVC and LVEDV. Our data provide genetic context to observational associations that earlier studies have reported [1-3], indicating a relationship between airflow obstruction and LV filling in the general population. Importantly, we found no evidence for a reverse-causation phenomenon. To date, we are not aware of a MR study that directly evaluated the effect of airflow obstruction on LV filling.

Several mechanisms of impaired LV filling with increasing airflow obstruction can be considered. Loss of pulmonary vasculature can occur even without manifest lung disease and the total volume of the pulmonary vasculature was associated with reduced cardiac filling [21]. Furthermore, pulmonary hyperinflation, which is particularly evident in patients with chronic obstructive pulmonary disease (COPD), has been suggested to decrease intrathoracic blood flow and reduce LV preload [22-24]. Interestingly, randomised and placebo-controlled trials have shown that in patients with COPD and hyperinflation bronchodilators increased LVEDV with an inverse correlation between treatment-associated change in residual volume and LVEDV [25, 26]. Using dual bronchodilator (indacaterol and glycopyrronium) achieved an increase of LVEDV by approximate $10 \%$ [25]. Alveolar hypoxia, in part a consequence of ventilatory changes, can also occur under physiological conditions and triggers hypoxic pulmonary vasoconstriction [27]. If sustained this mechanism contributes to volume and pressure overload of the right ventricle (RV), which in the context of diastolic ventricular interdependence decreases LV volumes [28]. An increased pulmonary vascular resistance and RV afterload is well established in patients with COPD and is in general dependent on the severity of the disease [29].

Assessing the individual SNPs in relation to the overall estimate could elucidate molecular underpinnings of the observed causal relationship between FEV1/ FVC and LVEDV. Nineteen SNPs exhibited a significant effect in single SNP MR and shared the effect direction of the overall result. The top three SNPs with 


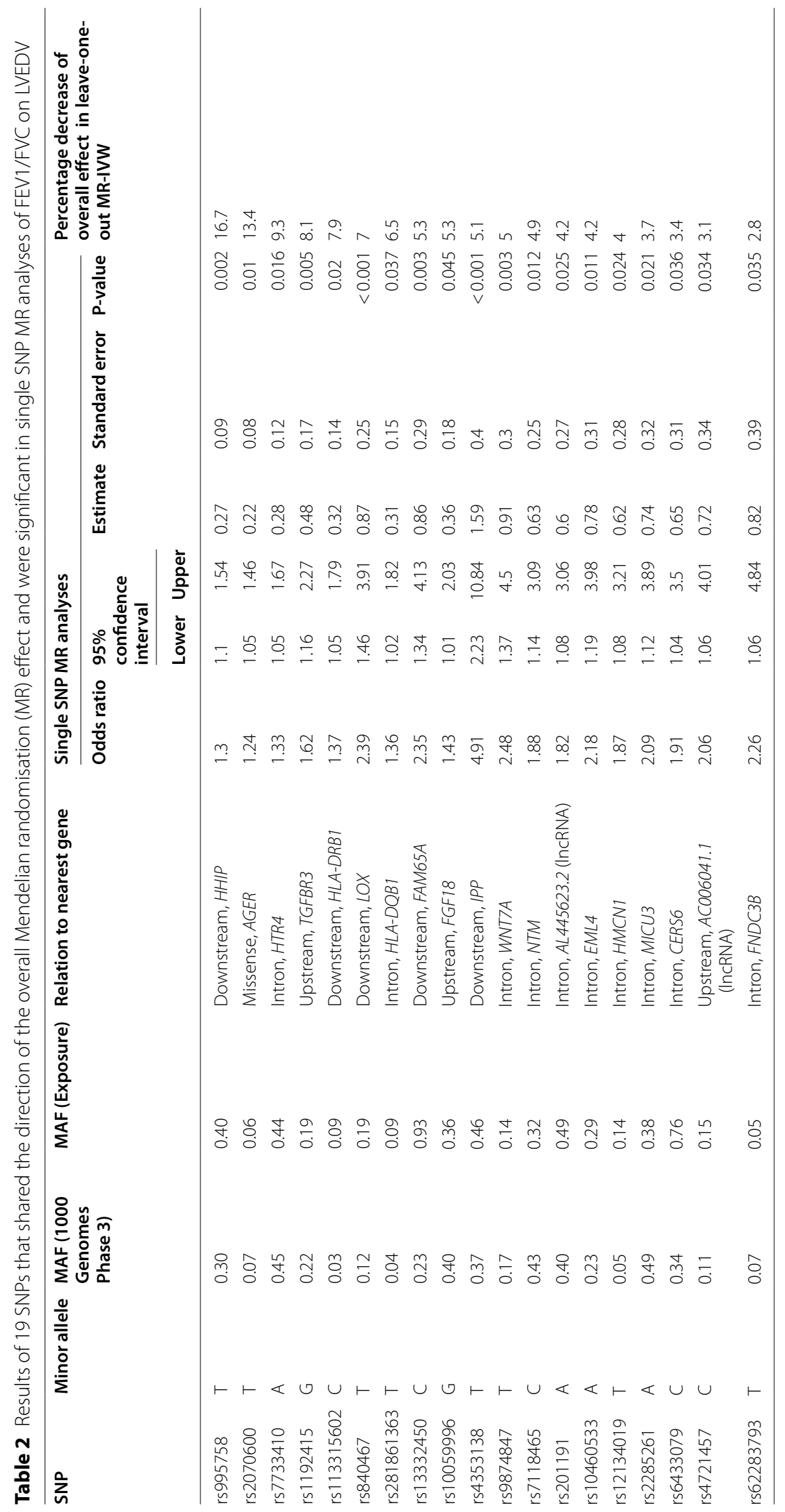


major contribution to the overall effect were rs 995758 (chr4:145,478,201-C-T), rs2070600 (chr6:32,151,443C-T) and rs7733410 (chr5:147,856,522-G-A). When leaving one of these three SNPs out, the effect of the overall MR-IVW was reduced by $17 \%, 13 \%$ and $9 \%$, respectively. These loci have not only been linked to pulmonary function parameters such as FEV1/FVC [7], but also to COPD phenotypes including emphysema [30] and airway remodeling [31]. Rs995758 is located intergenic and the nearest gene, HHIP, encodes for the hedgehog interacting protein (HHIP). Mice with heterozygous HHIP knock-out $\left(H H I P^{+-}\right)$spontaneously develop airway remodeling with increased airway smooth muscle mass and increased airway resistance [32]. Rs2070600 is a missense variant in the AGER gene encoding for the type I receptor for advanced glycation end-products (RAGE). This variant is predicted to be tolerable (raw combined annotation dependent depletion [CADD] score of 3.5) but influences protein abundance [33, 34]. Protein expression of RAGE in airway cells was also implicated in sensitization after pulmonary allergen exposure and with immune regulatory function at a later stage of asthmatic disease [35]. Interestingly, variation at rs2070600 associated with outcomes in heart failure patients but not with risk of heart failure in a Chinese population [36]. The soluble form of RAGE (sRAGE) can be measured in the circulation and lower levels could serve as a biomarker for airflow obstruction [34], while levels seem to be elevated in patients with heart failure, indicating that interpretation of this biomarker in the context of cardiopulmonary interaction remains unclear [37]. The variant rs7733410 is located intronic to the HTR4 gene encoding the 5-hydroxytryptamine $(5-\mathrm{HT})$ /serotonin receptor 4 (HTR4). Mice deficient for this protein $\left(H T R 4^{-1-}\right)$ were found to have increased airway resistance and exhibit increased methacholine-induced airway hyperresponsiveness [38]. Since HTR4 is temporally expressed in developing human lungs, it could potentially be implicated in lung development and physiological cardiopulmonary interaction [39].

We acknowledge as limitation that we cannot fully exclude residual confounders. Common diseases or risk factors potentially affecting FEV1/FVC and/or LVEDV could be present in a subgroup of individuals from the general population and could provoke a systematic bias, particularly in settings when pleiotropic effects of multiple genetic variants act through the same confounder (i.e., violated InSIDE assumption). By excluding all variants with known GWAS signals in a sensitvity analysis, we sought to control for this scenario. In addition, overlapping datasets for the exposure and outcome variable could cause potential overfitting. In a sensitivity analysis, we therefore selected variants associated with FEV1/FVC in the smaller subpopulation of the GWAS meta-analysis (SpiroMeta consortium population) [14].

\section{Conclusion}

In summary, our present study provides genetic evidence for a causal link between airflow obstruction and LV filling by investigating the largest-to-date GWAS on FEV1/ FVC and LVEDV in the general population. Our results suggest that even subclinical reduction in airflow impairs LV function, and that targeting airflow obstruction can lead to direct cardiac improvements, demonstrated by an increase in LVEDV. Functional annotation of single SNPs contributing most to the causal effect estimate could help to prioritise biological pathways for biomarker-discovery and therapeutic intervention.

\begin{abstract}
Abbreviations
CARDIA: Coronary Artery Risk Development in Young Adults Study; CCHS: Copenhagen City Heart Study; Cl: Confidence interval; COPD: Chronic obstructive pulmonary disease; FEV1: Forced expiratory volume in $1 \mathrm{~s}$; FVC: Forced vital capacity; GWAS: Genome-wide association studies; HHIP: Hedgehog interacting protein; HTR4: 5-hydroxytryptamine receptor 4; InSIDE: Instrument Strength Independent of Direct Effect; IV: Instrument variable(s); IVW: Inverse variance weighted; LD: Linkage disequilibrium; LV: Left ventricular; LVEDV: LV end-diastolic volume; MAF: Minor allele frequency; MESA: Multi-Ethnic Study of Atherosclerosis Lung Study; MR: Mendelian randomisation; NOME: No measurement error; OR: Odds ratio; PRESSO: Pleiotropy RESidual Sum and Outlier; RAGE: Receptor for advanced glycation end-products; RV: Right ventricle; SNP: Single-nucleotide polymorphism; UK: United Kingdom; WM: Weighted median.
\end{abstract}

\section{Supplementary Information}

The online version contains supplementary material available at https://doi. org/10.1186/s12931-021-01795-9.

Additional file 1: Fig. S1. Variants filtering steps applied. Blue boxes indicate the set of IV used to estimate the causal effect. Fig. S2. Funnel plots on the three different IVs used to estimate the causal effect. Point estimate from IVW and MR-Egger displayed.

Additional file 2: Table S1. Detailed information on the IV used in the MR analyses. Table S2. Intercept from Egger-regression analyses. Table S3. Results from single SNP MR based on the ratio estimate and from leaveone-out MR based on the IVW method. Table S4. Association of the IV with potential covariates retrieved from a curated database of human genotype-phenotype associations. P-value of association displayed.

\section{Acknowledgements}

Not applicable.

\section{Authors' contributions}

LH carried out the statistical analysis and drafted the manuscript. JKH, MS, TO, HW and HK participated in the design of the study, interpretation of findings, and finishing the manuscript draft. All authors read and approved the final version of the manuscript.

\section{Funding}

Open Access funding enabled and organized by Projekt DEAL. This research received no specific grant from any funding agency in the public, commercial, or not-for-profit sectors. 


\section{Availability of data and materials}

The datasets and bioinformatical tools supporting the conclusions of this article are publicly available $[5,7,16]$.

\section{Declarations}

\section{Ethics approval and consent to participate}

UK Biobank and Spirometa consortium have approval from local research ethics committees and/or institutional review boards. All participants in these studies provided written informed consent.

\section{Consent for publication}

Individual persons data were not reported.

\section{Competing interests}

All authors take responsibility for all aspects of the reliability and freedom from bias of the data presented and their discussed interpretation. No potential conflict of interest was declared by the authors that may potentially influence the writing of the manuscript.

\section{Author details}

1 Abteilung für Pneumologie, Centrum für Pulmonal Arterielle Hypertonie Hamburg (CPAHH), Universitätsklinikum Hamburg-Eppendorf, Hamburg, Germany. ${ }^{2}$ Pneumologische Forschungsinstitut an der LungenClinic Grosshansdorf, Airway Research Center North (ARCN), German Center for Lung Research (DZL), Grosshansdorf, Germany.

\section{Received: 4 March 2021 Accepted: 30 June 2021}

Published online: 07 July 2021

\section{References}

1. Barr RG, Bluemke DA, Ahmed FS, Carr JJ, Enright PL, Hoffman EA, Jiang R, Kawut SM, Kronmal RA, Lima JA, et al. Percent emphysema, airflow obstruction, and impaired left ventricular filling. N Engl J Med. 2010;362:217-27.

2. Cuttica MJ, Colangelo LA, Shah SJ, Lima J, Kishi S, Arynchyn A, Jacobs DR Jr, Thyagarajan B, Liu K, Lloyd-Jones D, Kalhan R. Loss of lung health from young adulthood and cardiac phenotypes in middle age. Am J Respir Crit Care Med. 2015;192:76-85.

3. Baum C, Ojeda FM, Wild PS, Rzayeva N, Zeller T, Sinning CR, Pfeiffer N, Beutel M, Blettner M, Lackner KJ, et al. Subclinical impairment of lung function is related to mild cardiac dysfunction and manifest heart failure in the general population. Int J Cardiol. 2016;218:298-304.

4. Lange P, Nyboe J, Jensen G, Schnohr P, Appleyard M. Ventilatory function impairment and risk of cardiovascular death and of fatal or non-fatal myocardial infarction. Eur Respir J. 1991;4:1080-7.

5. Pirruccello JP, Bick A, Wang M, Chaffin M, Friedman S, Yao J, Guo X Venkatesh BA, Taylor KD, Post WS, et al. Analysis of cardiac magnetic resonance imaging in 36,000 individuals yields genetic insights into dilated cardiomyopathy. Nat Commun. 2020;11:2254.

6. Aung N, Vargas JD, Yang C, Cabrera CP, Warren HR, Fung K, Tzanis E, Barnes MR, Rotter JI, Taylor KD, et al. Genome-wide analysis of left ventricular image-derived phenotypes identifies fourteen loci associated with cardiac morphogenesis and heart failure development. Circulation. 2019:140:1318-30.

7. Shrine N, Guyatt AL, Erzurumluoglu AM, Jackson VE, Hobbs BD, Melbourne CA, Batini C, Fawcett KA, Song K, Sakornsakolpat P, et al. New genetic signals for lung function highlight pathways and chronic obstructive pulmonary disease associations across multiple ancestries. Nat Genet. 2019:51:481-93.

8. Zhao X, Qiao D, Yang C, Kasela S, Kim W, Ma Y, Shrine N, Batini C, Sofer T, Taliun SAG, et al. Whole genome sequence analysis of pulmonary function and COPD in 19,996 multi-ethnic participants. Nat Commun. 2020;11:5182.

9. Smith GD, Ebrahim S. "Mendelian randomization": can genetic epidemiology contribute to understanding environmental determinants of disease? Int J Epidemiol. 2003;32:1-22.
10. Lawlor DA. Commentary: Two-sample Mendelian randomization: opportunities and challenges. Int J Epidemiol. 2016;45:908-15.

11. Pulit SL, de With SA, de Bakker PI. Resetting the bar: Statistical significance in whole-genome sequencing-based association studies of global populations. Genet Epidemiol. 2017:41:145-51.

12. Kamat MA, Blackshaw JA, Young R, Surendran P, Burgess S, Danesh J, Butterworth AS, Staley JR. PhenoScanner V2: an expanded tool for searching human genotype-phenotype associations. Bioinformatics. 2019;35:4851-3.

13. Pierce $B L$, Burgess $S$. Efficient design for Mendelian randomization studies: subsample and 2-sample instrumental variable estimators. Am J Epidemiol. 2013;178:1177-84.

14. Soler Artigas M, Wain LV, Miller S, Kheirallah AK, Huffman JE, Ntalla I, Shrine N, Obeidat M, Trochet H, McArdle WL, et al. Sixteen new lung function signals identified through 1000 Genomes Project reference panel imputation. Nat Commun. 2015:6:8658.

15. Burgess S, Davey Smith G, Davies NM, Dudbridge F, Gill D, Glymour MM Hartwig FP, Holmes MV, Minelli C, Relton CL, Theodoratou E. Guidelines for performing Mendelian randomization investigations. Wellcome Open Res. 2019:4:186.

16. Hemani G, Zheng J, Elsworth B, Wade KH, Haberland V, Baird D, Laurin C, Burgess S, Bowden J, Langdon R, et al. The MR-Base platform supports systematic causal inference across the human phenome. Elife. 2018;7:1.

17. Bowden J, Davey Smith G, Burgess S. Mendelian randomization with invalid instruments: effect estimation and bias detection through Egger regression. Int J Epidemiol. 2015;44:512-25.

18. Bowden J, Davey Smith G, Haycock PC, Burgess S. Consistent estimation in Mendelian randomization with some invalid instruments using a weighted median estimator. Genet Epidemiol. 2016:40:304-14.

19. Bowden J, Del Greco MF, Minelli C, Davey Smith G, Sheehan NA, Thompson JR. Assessing the suitability of summary data for two-sample Mendelian randomization analyses using MR-Egger regression: the role of the $\mathrm{I}^{2}$ statistic. Int J Epidemiol. 2016:45:1961-74.

20. Verbanck M, Chen CY, Neale B, Do R. Detection of widespread horizontal pleiotropy in causal relationships inferred from Mendelian randomization between complex traits and diseases. Nat Genet. 2018;50:693-8.

21. Aaron CP, Hoffman EA, Lima JAC, Kawut SM, Bertoni AG, Vogel-Claussen J, Habibi M, Hueper K, Jacobs DR Jr, Kalhan R, et al. Pulmonary vascular volume, impaired left ventricular filling and dyspnea: The MESA Lung Study. PLoS ONE. 2017;12:e0176180.

22. Watz H. On trapped air and trapped blood in chronic obstructive pulmonary disease. Am J Respir Crit Care Med. 2019;199:1047-8.

23. Watz H, Waschki B, Meyer T, Kretschmar G, Kirsten A, Claussen M, Magnussen $\mathrm{H}$. Decreasing cardiac chamber sizes and associated heart dysfunction in COPD: role of hyperinflation. Chest. 2010;138:32-8.

24. Alter P, Jörres RA, Watz H, Welte T, Gläser S, Schulz H, Bals R, Karch A, Wouters EFM, Vestbo J, et al. Left ventricular volume and wall stress are linked to lung function impairment in COPD. Int J Cardiol. 2018;261:172-8

25. Hohlfeld JM, Vogel-Claussen J, Biller H, Berliner D, Berschneider K, Tillmann HC, Hiltl S, Bauersachs J, Welte T. Effect of lung deflation with indacaterol plus glycopyrronium on ventricular filling in patients with hyperinflation and COPD (CLAIM): a double-blind, randomised, crossover, placebo-controlled, single-centre trial. Lancet Respir Med. 2018:6·368-78.

26. Stone IS, Barnes NC, James WY, Midwinter D, Boubertakh R, Follows R, John L, Petersen SE. Lung deflation and cardiovascular structure and function in chronic obstructive pulmonary disease. A randomized controlled trial. Am J Respir Crit Care Med. 2016;193:717-26.

27. Sommer N, Strielkov I, Pak O, Weissmann N. Oxygen sensing and signal transduction in hypoxic pulmonary vasoconstriction. Eur Respir J. 2016:47:288-303

28. Naeije R, Badagliacca R. The overloaded right heart and ventricular interdependence. Cardiovasc Res. 2017;113:1474-85.

29. Nathan SD, Barbera JA, Gaine SP, Harari S, Martinez FJ, Olschewski H, Olsson KM, Peacock AJ, Pepke-Zaba J, Provencher S, et al. Pulmonary hypertension in chronic lung disease and hypoxia. Eur Respir J. 2019;53:1.

30. Cho MH, Castaldi PJ, Hersh CP, Hobbs BD, Barr RG, Tal-Singer R, Bakke P, Gulsvik A, San José Estépar R, Van Beek EJ, et al. A genome-wide association study of emphysema and airway quantitative imaging phenotypes. Am J Respir Crit Care Med. 2015;192:559-69.

31. van der Plaat DA, de Jong K, Lahousse L, Faiz A, Vonk JM, van Diemen CC, Nedeljkovic I, Amin N, Obeidat M, van Duijn CM, et al. The well-known 
gene HHIP and novel gene MECR are implicated in small airway obstruction. Am J Respir Crit Care Med. 2016;194:1299-302.

32. Li Y, Zhang L, Polverino F, Guo F, Hao Y, Lao T, Xu S, Li L, Pham B, Owen CA, Zhou X. Hedgehog interacting protein (HHIP) represses airway remodeling and metabolic reprogramming in COPD-derived airway smooth muscle cells. Sci Rep. 2021;11:9074.

33. Folkersen L, Fauman E, Sabater-Lleal M, Strawbridge RJ, Frånberg M, Sennblad B, Baldassarre D, Veglia F, Humphries SE, Rauramaa R, et al. Mapping of 79 loci for 83 plasma protein biomarkers in cardiovascular disease. PLoS Genet. 2017;13:e1006706.

34. Pratte KA, Curtis JL, Kechris K, Couper D, Cho MH, Silverman EK, DeMeo DL, Sciurba FC, Zhang Y, Ortega VE, et al. Soluble receptor for advanced glycation end products (SRAGE) as a biomarker of COPD. Respir Res. 2021;22:127.

35. Perkins TN, Oczypok EA, Dutz RE, Donnell ML, Myerburg MM, Oury TD. The receptor for advanced glycation end products is a critical mediator of type 2 cytokine signaling in the lungs. J Allergy Clin Immunol. 2019;144:796-808.e712.
36. Li S, Hu D, Hu S, Sun Y, Zhang Y, Li H, Chen Y, Liu H, Cui G, Wang DW. Association of rs 2070600 in advanced glycosylation end-product specific receptor with prognosis of heart failure. ESC Heart Fail. 2020;7:3561-72.

37. Ahmed A, Ahmed S, Arvidsson M, Bouzina H, Lundgren J, Rådegran G. Elevated plasma sRAGE and IGFBP7 in heart failure decrease after heart transplantation in association with haemodynamics. ESC Heart Fail. 2020;7:2340-53.

38. House JS, Li H, DeGraff LM, Flake G, Zeldin DC, London SJ. Genetic variation in HTR4 and lung function: GWAS follow-up in mouse. Faseb J. 2015;29:323-35.

39. Hodge E, Nelson CP, Miller S, Billington CK, Stewart CE, Swan C, Malarstig A, Henry AP, Gowland C, Melén E, et al. HTR4 gene structure and altered expression in the developing lung. Respir Res. 2013;14:77.

\section{Publisher's Note}

Springer Nature remains neutral with regard to jurisdictional claims in published maps and institutional affiliations.
Ready to submit your research? Choose BMC and benefit from:

- fast, convenient online submission

- thorough peer review by experienced researchers in your field

- rapid publication on acceptance

- support for research data, including large and complex data types

- gold Open Access which fosters wider collaboration and increased citations

- maximum visibility for your research: over $100 \mathrm{M}$ website views per year

At $\mathrm{BMC}$, research is always in progress.

Learn more biomedcentral.com/submissions 